\title{
Self perceived work related stress and the relation with salivary lgA and lysozyme among emergency department nurses
}

\author{
Y Yang, D Koh, V Ng, C Y Lee, G Chan, F Dong, S H Goh, V Anantharaman, S E Chia
}

See end of article for authors' affiliations

.....................

Correspondence to: Professor D Koh, Department of Community, Occupational and Family Medicine (MD3), Faculty of Medicine, National

University of Singapore,

16, Medical Drive

Singapore 117597 ;

cofkohd@nus.edu.sg

Accepted 14 May 2002

\begin{abstract}
Aims: To assess and compare the self perceived work related stress among emergency department (ED) and general ward (GW) nurses, and to investigate its relation with salivary $\lg A$ and lysozyme. Methods: One hundred and thirty two of $208(63.5 \%)$ registered female ED and GW nurses participated in the study. A modified mental health professional stress scale (PSS) was used to measure self perceived stress. ELISA methods were used to determine the salivary lgA and lysozyme levels.

Results: On PSS, ED nurses had higher scores (mean 1.51) than GW nurses (1.30). The scores of PSS subscales such as organisational structure and processes (OS), lack of resources (RES), and conflict with other professionals (COF) were higher in ED than in GW nurses. ED nurses had lower secretion rates of $\operatorname{lgA}$ (geometric mean $(G M) 49.1 \mathrm{\mu g} / \mathrm{min}$ ) and lysozyme $(G M 20.0 \mu \mathrm{g} / \mathrm{min}$ ) than $\mathrm{GW}$ nurses 168.2 $\mathrm{\mu g} / \mathrm{min}, 30.5 \mu \mathrm{g} / \mathrm{min}$ ). Significant correlations were observed between PSS and $\log \lg A$ and lysozyme secretion rates. OS, RES, and COF were correlated with log lgA and lysozyme levels.

Conclusion: ED nurses, who reported a higher level of professional stress, showed significantly lower secretion rates of salivary $\lg A$ and lysozyme compared to GW nurses. Salivary lgA and lysozyme were inversely correlated with self perceived work related stress. As these salivary biomarkers are reflective of the mucosal immunity, results support the inverse relation between stress and mucosal immunity.
\end{abstract}

$\mathrm{R}$ esearch has shown that nursing is a stressful occupation..$^{12}$ Growing evidence suggests that it might be increasing in severity. ${ }^{3}$ Emergency department (ED) nursing is a special kind of nursing. In addition to the stressors that are encountered by nurses such as work overload, dealing with difficult patients, role conflict, lack of support, and home-work conflicts, ${ }^{45}$ ED nurses are in a position that is expected to deal with additional stressors. These include unexpected numbers of patients at any time, unexpected rapid changes in patients' situations, and response to distressing or traumatic incidents such as sudden death, patient violence, inappropriate attendees, and physical or verbal abuse on a daily basis. ${ }^{67}$ Emergency nursing is a highly stressful profession. ${ }^{8}$

It is believed that nurse stress could lead to a decrease in quality of care, increased staff turnover, and impairment of nurses' health. ' Studies that relate to nurse working stress within the specialty of ED unit have mostly been conducted using subjective questionnaire or interview methods. Furthermore, different questionnaires have been used in different studies, making it difficult to compare the study results. Studies have mainly been carried out in the USA, UK, and Canada. ${ }^{8}$

Recently, various correlational and longitudinal studies have lent support to the hypothesis that chronic psychological stress could lead to impairment of immune function, leaving the individuals exposed to greater risk of infection and illness. ${ }^{10-12}$ Secretory immunoglobulin A (sIgA) in saliva, the main immunological defence of mucosal surfaces, had repeatedly been shown to be sensitive to psychological variables. It is believed to be representative of functional status of the entire mucosal immune system. ${ }^{13}$

Lysozyme, or muramidase, one of the major nonimmunological antimicrobial proteins in saliva, is widely distributed in human tissues and secretions. ${ }^{14}$ It is considered to belong to a primitive defence system, known as the innate immune system. Lysozyme is a cationic protein with wide antimicrobial activities. The mechanism of the antimicrobial function of lysozyme is through its enzymatic activity, which cleaves $(-1,4)$ glycosidic bonds between muramic acid and $\mathrm{N}$-acetylglucosamine residues in the peptidoglycan of the bacterial cell wall. ${ }^{15}{ }^{16}$ Other proposed antimicrobial activities of lysozyme include inhibition of bacterial growth, metabolism, and dechaining. ${ }^{17}$

The relation between stress and salivary lysozyme is not clear. Recently, Perera et al assessed the relation between stress and salivary lysozyme in two studies. One study showed a decreased salivary lysozyme after academic examinations in 39 students. $^{18}$ The other study, on 15 students, showed increased salivary lysozyme level after exposure to various relaxation strategies. ${ }^{19}$ However, Ng et al found nosignificant correlation between the self perceived stress levels and salivary lysozyme in 124 female Singapore nurses. ${ }^{20}$ Their study utilised a general stress questionnaire that focused on the average Asian population, and was not specific for nurses. In all the above mentioned studies, lysozyme was measured using a lysoplate method, which is thought to be less sensitive than the enzyme linked immunosorbent assay (ELISA) method. However, significantly different levels of salivary lysozyme were found in different nursing units, similar to those obtained with IgA. ${ }^{20}$ A close linkage between salivary lysozyme and sIgA has also been suggested in students, nurses, and patients with psoriasis. ${ }^{19-21}$

The objectives of this study were: (1) to assess and compare the self perceived work related stress of female ED and general ward (GW) nurses; and (2) to investigate the relation between salivary IgA and lysozyme with self perceived work related stress.

Abbreviations: ED, emergency department; GM, geometric mean; GW, general ward; PBS, phosphate buffered saline; PSS, professional stress scale; slgA, secretory immunoglobulin A; URTI, upper respiratory tract infection 


\section{METHODS \\ Subjects}

This cross sectional study was conducted in the ED departments of three big public hospitals in Singapore. There were 142 eligible female nurses employed in these ED departments. In one of the hospitals, 66 eligible female nurses in three general wards were chosen as a control group. Participation was voluntary and anonymous. The objectives of the study were explained to the nursing officers and the nurses of the departments involved. The nursing officers helped to deliver the questionnaires and reminded nurses about filling in the questionnaire. In order to maintain confidentiality, the completed questionnaires were sealed before being handed to the nursing officers.

\section{Questionnaire}

The self administered questionnaire included items of information on sociodemographic characteristics such as age, marital status, educational level, nursing appointment, and years of working experience in their respective departments.

\section{Mental health professionals stress scale (PSS)}

A questionnaire developed by Cushway et al was used to assess the self perceived work related stress for mental health professionals. It included almost all the stressors encountered by the nursing profession, even in ED nursing. With some modifications to those questions that were unique to mental health professionals, it was used to assess the work related stress of ED nurses in this study. The questionnaire included seven parts with 42 items. Nurses were asked to indicate on a four point scale how frequently they experienced such stressful situations in the present department. The score was from 0 (does not apply to me) to 3 (does apply to me) (table 1). As suggested by the author, the total score was obtained by summing all the scores and dividing by the total number of questions to derive the average. Internal consistency coefficients for these scales range from 0.87 to 0.94 . The concurrent validity of the questionnaire is good; there is a good relation with criterion measures such as general health questionnaire and symptom checklist. However, assessment of test-retest reliability and further validation is needed, as this is a relatively newly developed instrument. ${ }^{22}$

\section{Saliva collection}

A written protocol on how to collect the saliva was given to the nurses. In addition, they were also briefed on the collection method and supervised on the day of collection. A single timed five minute unstimulated total saliva was obtained from nurses who participated in the study when they were on their morning shift. The time of saliva collection was between $1 \mathrm{pm}$ and $3 \mathrm{pm}$, after they finished their work. This timing was designed to minimise the variation that might be introduced by circadian rhythm.

Nurses were asked not to eat and drink (except water) one hour prior to saliva collection, as food debris could stimulate salivation. Nurses were also asked to record whether they had suffered from upper respiratory tract infection (URTI) on the day the saliva was collected or in the past week. After collection, the volume of saliva was measured and divided by five minutes to obtain the salivary flow rate, expressed in $\mathrm{ml} / \mathrm{min}$. The samples were immediately taken to the laboratory and stored frozen at $-70^{\circ} \mathrm{C}$ until required for assay.

\section{Valid subjects}

To ensure validity of the results, exclusion criteria for participation were: pregnancy; chronic diseases affecting the immune system; regular medication with known effects on the immune system; and present or past (one week) history of URTI, as URTI will affect the level of IgA and lysozyme in the saliva.
Table 1 Modified mental health professional stress scale (PSS)

Subjects are asked to indicate on a four point scale how frequently they experienced such situations as stressful in the present department*

\section{Work load (WL)}

A. Too much work to do

B. Too many different things to do

C. Not enough time to complete all tasks satisfactorily

D. Too many patients

E. Too long working hours

F. Not enough time for recreation

2. Patient related difficulties (PD)

A. Communicating with patients

B. Dealing with death or suffering

C. No or slowness of improvement in patient's situation

D. Dealing with difficult or demanding patients

E. Dealing with physically threatening patients

F. Managing therapeutic relations with patients

3. Organisational structure and processes (OS)

A. Lack of support from management

B. Lack of relationship with your immediate supervisors

C. Lack of communications and flow of information at work

D. Poor management and supervision

E. Lack of ways that conflicts are resolved in the organisation

F. Poor organisational structure and policies

4. Lack of resources (RES)

A. Lack of adequate staffing

B. Lack of financial resources for training courses/workshops

C. Lack of adequate equipment/supplies

D. Lack of adequate protection in potentially dangerous environment

E. Inadequate clerical/technical back up

F. Poor physical working conditions

5. Conflict with other professionals (COF)

A. Conflict with other professionals (e.g. doctor)

B. Conflicting roles with other health professionals

C. Conflict with staff in other departments

D. Criticism by other professionals (e.g. doctor)

E. Lack of emotional support from colleagues

F. Difficulty of working with certain colleagues

6. Professional self doubt (SD)

A. Feeling inadequately skilled for dealing with emotional needs of patients

B. Feeling uncertain about own capabilities

C. Feeling inadequately skilled for dealing with difficult patients

D. Feeling about the efficacy of therapeutic endeavours

E. Difficulty in keeping professional/clinical skills up to date

F. Fear of a mistake over a patient's treatment

7. Home-work conflicts

A. Not enough time with friends

B. Inability to separate personal from professional role

C. Taking work home

D. Relationship with spouse/partner affects work

E. Work results in feelings of emptiness and/or isolation

F. Inadequate time for friendships/social relationships

${ }^{*} 0$, does not apply to me; 1 , does not usually apply to me; 2 , does occasionally apply to me; 3 , does apply to me.

Modified from Cushway et al. ${ }^{22}$

Furthermore, the flow rate of saliva of valid subjects had to be at least $0.1 \mathrm{ml} / \mathrm{min}$ (under basal conditions, the rate of saliva production is $0.5 \mathrm{ml} / \mathrm{min}) .{ }^{23}$ Subjects with a flow rate of less than $0.1 \mathrm{ml} / \mathrm{min}$ would probably not have collected the saliva properly for the five minute period.

\section{Laboratory methods and analyses}

An ELISA method was used to determine the salivary IgA concentration $(\mu \mathrm{g} / \mathrm{ml}){ }^{24}{ }^{25}$ The salivary lysozyme concentration was measured using an ELISA method that was developed in our laboratory. In brief, a 96 well microtitre ELISA plate was coated overnight at $4^{\circ} \mathrm{C}$ with $200 \mu \mathrm{l}$ of rabbit antihuman lysozyme at a concentration of $7.0 \mathrm{mg} / \mathrm{l}$ in sodium carbonate-bicarbonate buffer ( $\mathrm{pH}$ 9.6). The well was washed with phosphate buffered saline (PBS) containing $0.05 \%(\mathrm{v} / \mathrm{v})$ Tween 20 (PBS/Tween), and blot dried. The plate was used 
Table 2 Demographic characteristics and work experience of emergency department (ED) and general ward (GW) nurses

\begin{tabular}{|c|c|c|c|c|}
\hline Characteristic & $\begin{array}{l}\text { ED nurses } \\
\mathrm{n}=82\end{array}$ & $\begin{array}{l}\text { GW nurses } \\
n=50\end{array}$ & ED-GW $(95 \% \mathrm{Cl})$ & $\begin{array}{l}\text { Total } \\
n=132\end{array}$ \\
\hline \multicolumn{5}{|l|}{ Age (years)* } \\
\hline Mean & 32.1 & 27.6 & \multirow[t]{3}{*}{4.5 (3.2 to 7.7$)$} & 30 \\
\hline SD & 8.8 & 4.5 & & 7.9 \\
\hline Min-max & $21-56$ & $21-48$ & & $21-56$ \\
\hline \multicolumn{5}{|l|}{ Marital status $(\%) \dagger$} \\
\hline Married & 45 & 24 & $21 \quad$ (5 to 37$)$ & 37 \\
\hline \multicolumn{5}{|l|}{ Education $(\%) \ddagger$} \\
\hline Higher than diploma & 52 & 44 & $8 \quad(-9$ to 25$)$ & 49 \\
\hline Diploma & 30 & 34 & $-4 \quad(-12$ to 20$)$ & 32 \\
\hline Secondary & 18 & 22 & $-4 \quad(-10$ to 18$)$ & 19 \\
\hline \multicolumn{5}{|c|}{ Work experience (years) ** } \\
\hline Mean & 5.3 & 2.9 & \multirow[t]{3}{*}{$2.4(1.2$ to 3.5$)$} & 4.4 \\
\hline SD & 4.2 & 2.2 & & 3.8 \\
\hline Min-max & $0.5-15$ & $1.0-12$ & & $0.5-15$ \\
\hline
\end{tabular}

immediately, or stored at $-20^{\circ} \mathrm{C}$ for up to two months. A $200 \mu \mathrm{l}$ aliquot of $2 \%(\mathrm{w} / \mathrm{v})$ bovine serum albumin (radioimmunoassay grade, Sigma, USA) in PBS/Tween was added and left at room temperature for 120 minutes. The well was washed with PBS/Tween 20; $100 \mu \mathrm{l}$ of specimen diluted in PBS (1/50) and $100 \mu \mathrm{l}$ biotinylated lysozyme (1/1000) (radioimmunoassay grade, Sigma, USA) was added to each well. The plate was incubated at $37^{\circ} \mathrm{C}$ for 60 minutes (standard at 50, 25, 12.5, $6.25,3.125 \mu \mathrm{g} / \mathrm{ml}$; biotinylated lysozyme $1 / 1000$ ). The well was washed with PBS/Tween 20; $200 \mu \mathrm{l}$ avidin alkaline phosphatase diluted in 1/3000 in PBS was added and incubated at $37^{\circ} \mathrm{C}$ for 60 minutes. After washing, $200 \mu \mathrm{l}$ of enzyme substrate p-nitrophenyl phosphate sodium $(0.1 \% \mathrm{w} / \mathrm{v})$ in dietheanolamine buffer (DEA buffer, $\mathrm{pH}$ 9.8) was added and incubated for 30 minutes at room temperature (kept in the dark). The reaction was stopped with $50 \mu \mathrm{l} 1.0 \mathrm{M} \mathrm{NaOH}$. The absorbance was read on a Bio-Rad microplate reader at 405 $\mathrm{nm}$. Each run included five twofold dilutions of purified human urine lysozyme (stock concentration 50 mg/l; Sigma Chemical Co., St Louis, MO) from which a standard curve was generated. The salivary IgA and lysozyme secretion rate $(\mu \mathrm{g} / \mathrm{min})$ were computed by multiplying the absolute IgA and lysozyme concentration with the absolute saliva flow rate.

\section{Statistical analysis}

Data are presented as mean (SD); all p values and confidence intervals are two sided, and the level of statistical significance was considered to be $\mathrm{p}<0.05$. Two sample independent $t$ tests were used in the univariate analysis. General linear models were used in the multivariate analysis for controlling the possible effect of marital status and work experience, as marital status and work experience might affect the level of work related stress. Partial correlations between stress scores and biomarkers were calculated after controlling for marital status and work experience. A Bonferroni correction was made to control for type 1 error inflation. As the data of salivary IgA and lysozyme showed positively skewed distributions, logarithmic transformations were performed for all data for further statistical analyses. The data analyses were performed with SPSS software. ${ }^{26}$

\section{RESULTS}

\section{Response rate}

One hundred and sixty two nurses of 208 eligible nurses took part in this study, of which 106 of $142(74.6 \%)$ ED nurses and 56 of $66(84.8 \%)$ GW nurses completed a questionnaire and provided a salivary sample. The overall response rate was $77.9 \%$.
Eventually 132 nurses who did not have URTI and who had properly collected saliva samples were selected for further analyses, 82 in the ED and 50 in the GW nurse group. This represents an overall response rate of $63.5 \%$. Of the excluded nurses, 16 had a salivary flow rate of less than $0.1 \mathrm{ml} / \mathrm{min}$, and 13 had suffered from an URTI either currently or during the past week. One nurse was excluded because of pregnancy. Comparisons between the 132 participants and the 30 subjects who dropped out of the study revealed no significant differences with respect to demographic or psychological variables.

\section{Sociodemographic characteristics}

Among the 132 selected nurses, most (85.6\%) were 20-40 years old. The ED nurses were slightly older (mean age 32.1 years) compared to the GW nurses (27.6 years) $(p=0.002)$. Eighty per cent of the nurses had obtained a nursing diploma or higher; the others had at least completed secondary school. Forty three per cent of nurses in ED were married, as were 22\% of nurses in GW $(p=0.016)$. ED nurses had slightly more working experience ( 5.3 years) than GW nurses (2.9 years) $(p=0.000)$. Marital status and working experience were used as covariates to adjust for their possible effects. There were no obvious differences in educational level and other factors between the two groups. Table 2 shows the sociodemographic characteristics.

\section{Self perceived work related stress}

The score of PSS of ED nurses (mean 1.51) was higher than that of GW nurses (1.30) $(\mathrm{p}=0.012)$. The difference was still observed after adjustment for marital status and working experience $(p=0.029)$. After finding a difference between ED and GW nurses on the PSS, we compared the subscales of PSS. Scores of organisational structure and processes, lack of resources, and conflict with other professions were significantly higher in ED compared to GW nurses (all p $=0.002$ ). After adjustment for marital status and working experience, all three subscales and patient related difficulties showed significant differences (all $\mathrm{p}<0.01$ ). The other subscales showed no significant differences between ED and GW nurses. However, after Bonferroni adjustment, only organisational structure and processes showed significant differences (table 3).

\section{Statistical analyses of salivary biomarkers Salivary $\lg \mathrm{A}$}

SIgA concentration and secretion rate were lower in ED nurses (geometric mean (GM) 148.5, min-max 52.9-408.0 $\mu \mathrm{g} / \mathrm{ml}$; and 49.1, 5.4-47.7 $\mu \mathrm{g} / \mathrm{min}$ ) than that of GW nurses (GM 200.9, $\min -\max 54.3-95.9 \mu \mathrm{g} / \mathrm{ml}$; and 68.2, 29.8-220.1 $\mu \mathrm{g} / \mathrm{min}$ ) 
Table 3 Means, minimum to maximum (min-max), and 95\% Cl of group difference (ED-GW) of the scores of PSS and its subscales

\begin{tabular}{|c|c|c|c|c|c|c|c|}
\hline \multirow[b]{2}{*}{ Scales } & \multicolumn{2}{|c|}{$\begin{array}{l}\text { Emergency department } \\
\text { (ED) nurses }(n=82)\end{array}$} & \multicolumn{2}{|c|}{$\begin{array}{l}\text { General ward (GW) } \\
\text { nurses }(n=50)\end{array}$} & \multirow[b]{2}{*}{ ED-GW 95\% Cl } & \multirow[b]{2}{*}{$\mathrm{p}^{*}$} & \multirow[b]{2}{*}{ Adjusted $\mathrm{p}^{* *}$} \\
\hline & Mean & Min-max & Mean & Min-max & & & \\
\hline Mental health professional stress scale (PSS) & 1.51 & $0.0-3.0$ & 1.30 & $0.0-2.8$ & 0.05 to 0.38 & 0.012 & 0.029 \\
\hline Work load (WL) & 1.70 & $0.2-3.0$ & 1.61 & $0.0-2.7$ & -0.12 to0.30 & 0.389 & 0.395 \\
\hline Patient related difficulty (PD) & 1.82 & $0.5-3.0$ & 1.68 & $0.8-2.7$ & -0.12 to0.30 & 0.142 & 0.168 \\
\hline Organisational structure and processes (OS) & 1.54 & $0.0-3.0$ & 1.15 & $0.0-2.3$ & 0.15 to0.63 & $0.002 \dagger$ & $0.007 \dagger$ \\
\hline Lack of resources (RES) & 1.54 & $0.0-3.0$ & 1.13 & $0.0-2.3$ & 0.15 to 0.65 & $0.002 \dagger$ & 0.009 \\
\hline Conflict with other professionals (COF) & 1.46 & $0.0-3.0$ & 1.11 & $0.0-2.2$ & 0.13 to0.57 & $0.002 \dagger$ & 0.008 \\
\hline Professional self doubt (SD) & 1.40 & $0.0-3.0$ & 1.29 & $0.0-2.7$ & -0.13 to0.35 & 0.353 & 0.480 \\
\hline Home-work conflicts (HW) & 1.06 & $0.0-3.0$ & 1.12 & $0.0-2.8$ & -0.30 to0.17 & 0.598 & 0.587 \\
\hline
\end{tabular}

Table 4 Geometric means (GM), minimum to maximum (min-max), and 95\% Cl of group difference (ED-GW) for salivary lgA and lysozyme of emergency department (ED) and general ward (GW) nurses

\begin{tabular}{|c|c|c|c|c|c|c|c|}
\hline \multirow[b]{2}{*}{ Biomarkers } & \multicolumn{2}{|c|}{ ED nurse $(n=82)$} & \multicolumn{2}{|c|}{ GW nurse $(n=50)$} & \multirow[b]{2}{*}{ ED-GW 95\% Cl } & \multirow[b]{2}{*}{$\mathrm{p}^{*}$} & \multirow[b]{2}{*}{ Adjusted $\mathrm{p}^{* *}$} \\
\hline & GM & Min-max & GM & Min-max & & & \\
\hline \multicolumn{8}{|l|}{$\lg A$} \\
\hline Concentration $(\mu \mathrm{g} / \mathrm{ml})$ & 148.5 & $52.93-407.95$ & 200.9 & $54.28-495.90$ & -95.66 to-23.61 & $0.002 \dagger$ & $0.002 \dagger$ \\
\hline Secretion rate $(\mu \mathrm{g} / \mathrm{min})$ & 49.1 & $5.38-247.67$ & 68.2 & $29.84-220.13$ & -28.75 to-0.71 & $0.001 \dagger$ & 0.038 \\
\hline \multicolumn{8}{|l|}{ Lysozyme } \\
\hline Concentration ( $\mu \mathrm{g} / \mathrm{ml})$ & 62.4 & $12.92-307.45$ & 101.9 & $16.19-258.62$ & -59.74 to- 16.72 & $0.000 \dagger$ & $0.000 \dagger$ \\
\hline Secretion rate $(\mu \mathrm{g} / \mathrm{min})$ & 20.0 & $3.36-116.08$ & 30.5 & $4.63-117.04$ & -21.56 to -3.90 & $0.003 \dagger$ & $0.012 \dagger$ \\
\hline
\end{tabular}

\begin{tabular}{lll}
$\begin{array}{l}\text { Table } 5 \\
\text { Partial correlations between log salivary } \lg A \text { and lysozyme secretion rate } \\
\text { ( } \mathrm{g} / \mathrm{min}) \text { and scores of PSS and subscales }(\mathrm{n}=132)\end{array}$ \\
\hline & $\log \operatorname{lgA}(\mathrm{p})^{*}$ & Log lysozyme $(\mathrm{p})^{*}$ \\
\hline Mental health professional stress scale (PSS) & $-0.28(0.001)$ & $-0.28(0.001)$ \\
Workload (WL) & $-0.15(0.097)$ & $-0.08(0.338)$ \\
Patient related difficulty (PD) & $-0.15(0.094)$ & $-0.14(0.107)$ \\
Organisational structure (OS) & $-0.27(0.002) \dagger$ & $-0.20(0.023)$ \\
Lack of resource (RES) & $-0.24(0.005) \dagger$ & $-0.18(0.040)$ \\
Conflict with other professionals (COF) & $-0.28(0.001) \dagger$ & $-0.39(0.000) \dagger$ \\
Professional self doubt (SD) & $-0.07(0.464)$ & $-0.19(0.028)$ \\
Home-work conflict (HW) & $-0.33(0.000) \dagger$ & $-0.19(0.028)$ \\
\hline
\end{tabular}

*Partial correlations were calculated after controlling for marital status and work experience (years).

†Significant after Bonferroni correction for PSS subscales.

$(p=0.002,0.001)$. The differences still existed even after adjustment for marital status and working experience $(\mathrm{p}=0.002,0.038)$ (table 4). After Bonferroni correction, sIgA concentration still showed the difference between the two groups. Log salivary IgA secretion rate was negatively correlated with PSS $(r=-0.28, \mathrm{p}=0.001)$.

With regard to the subscales of PSS, organisational structure and processes, lack of resources, conflict with other professionals, and home-work conflict had significant negative correlations with $\log$ sIgA secretion rate $(r-0.24$ to -0.33 , all $\mathrm{p}<0.01)$. Other subscales had no significant correlations with log sIgA level. After Bonferroni correction, organisational structure and processes, lack of resources, and conflict with other professionals still showed significant correlations with $\log$ sIgA secretion rate (table 5 ).

\section{Salivary lysozyme}

Salivary lysozyme concentration and secretion rate were lower in ED nurses (GM 62.4, min-max 12.9-307.5 $\mu \mathrm{g} / \mathrm{ml}$; and 20.00,
3.4-116.1 $\mu \mathrm{g} / \mathrm{min})$ than that of GW nurses (101.9, 16.2-258.6 $\mu \mathrm{g} / \mathrm{ml}$; and 30.5, 4.6-117.0 $\mu \mathrm{g} / \mathrm{min})(\mathrm{p}=0.000,0.003)$. The differences could be observed even after adjustment for marital status and working experience $(p=0.000,0.012)$ (table 4$)$. After Bonferroni correction, salivary lysozyme concentration and secretion rate still showed difference between the two groups. Log salivary lysozyme secretion rate was negatively correlated with PSS $(r=-0.28, \mathrm{p}=0.001)$.

With regard to the subscales of PSS, organisational structure and processes, lack of resources, and conflict with other professionals, personal self doubt, and home-work conflict had significantly negative correlations with log salivary lysozyme secretion rate $(r-0.18$ to -0.39 , all $p<0.05)$. The other two subscales had no significant correlations (table 5). After Bonferroni correction, only conflict with other professionals still showed significant correlation with log salivary lysozyme level. Furthermore, log salivary lysozyme secretion rate was moderately correlated with that of $\operatorname{sigA}(r=0.35$, $\mathrm{p}=0.000$ ). 


\section{DISCUSSION}

Response rate

The response rate of $63.5 \%$ in this study was satisfactory, compared to other studies that had response rates of $27 \%{ }^{20}$ and $33 \% .{ }^{27}$ Only 16 of 162 nurses $(9.9 \%)$ were excluded from the study because of improper collection of salivary samples. No obvious difference in response rate was noted between ED $(74.6 \%)$ and GW (84.8\%) nurses.

\section{Sociodemographic characteristics}

One of the differences between ED and GW nurses in the sociodemographic characteristics was age. The mean difference was less than five years. Previous reports have indicated no significant age effect on salivary IgA and immunity in adults aged aged $15-70$ years. ${ }^{28}{ }^{29}$ Thus the age difference was unlikely to be a confounder. The second difference between ED and GW nurses was marital status. There were more married nurses in the ED (44\%) than in the GW (22\%) group. Fuhrer et al found that married women could get more social support from their family network, which could buffer the stress in their work. ${ }^{30}$ However, in a recent nursing stress study of 1043 Singapore nurses, Boey et al found that married nurses would generally have more housework to do, a common situation in Asia. ${ }^{5}$ Inability to meet the family demands because of work was highly detrimental to the nurses' emotional stability. ${ }^{5}$ Another difference was the working experience. ED nurses had slightly more working experience ( 5.3 years) than GW nurses (2.9 years). Again, in the study of Boey et al, nurses who had more than 10 years working experience reported significantly lower stress than the less experienced nurses. ${ }^{5}$ Thus, marital status and working experience were used as covariates to adjust for their possible effects.

\section{Self perceived work related stress}

ED nurses scored significantly higher on the PSS than GW nurses. This is in accordance with Revicki and Gershon's study results of work related stress in emergency medicine workers. ${ }^{31}$ The results indicate that nurses working in ED departments perceived themselves to experience more stress than GW nurses. From the PSS subscales, patient related difficulty and workload were the two subscales that showed the highest score for both groups. The result was similar to other study results ${ }^{52}$; even though no significant difference was shown between the two groups, ED nurses perceived higher levels of patient related difficulties, organisational structure, lack of resources, and conflict with other professionals than did GW nurses. This suggests that those subscales reflected important causes of work related stress.

The mean PSS score in this study was 1.51. The study of Cushway et al, study, where the mean PSS score of nurses was relatively low at 1.30, showed good internal consistency coefficients of 0.87 to $0.94 .^{22}$ Thus, PSS score within this range should have similar internal consistency.

\section{Salivary IgA}

ED nurses, who perceived a higher level of work related stress, had a significantly lower sIgA concentration and secretion rate than that of GW nurses. SIgA secretion rate was significantly negatively correlated with PSS. These results confirmed the findings of other studies. ${ }^{13} 202433$ SIgA was also significantly negatively correlated with the scores of organisational structure and process, lack of resources, conflict with other professionals, and home-work conflict. This is in line with the results that showed the differences between ED and GW nurses in answering the PSS. Furthermore, although homework conflict showed no difference between ED and GW nurses, home-work conflict was still very important in the generation of work related stress, as Asian women are the main force of domestic work. In the study of nurses by Boey et al, meeting family demands was significantly related to

\section{Main messages}

- Emergency department nurses experience more work related stress than general ward nurses.

- Organisational structure and processes, lack of resources, and conflict with other professions are important causes of nurse work related stress.

- Work related stress is negatively correlated with salivary $\lg \mathrm{A}$ and lysozyme levels, reflecting mucosal immunity.

anxiety and depression $(r=0.44, \mathrm{p}<0.001)$ and sense of inadequacy $(r=0.34, \mathrm{p}<0.01)$. This could be the reason for the negative correlation between sIgA and the score of homework conflict.

\section{Salivary lysozyme}

In this study, ED nurses who reported a higher level of work related stress had a significantly lower level of salivary lysozyme than GW nurses. The significant negative relation between salivary lysozyme secretion rate and stress level on the PSS was also observed in the studies by Perera and colleagues. ${ }^{18}{ }^{19}$ Furthermore, a positive correlation was found between sIgA and lysozyme secretion rate in all nurses $(r=0.35, \mathrm{p}=0.000)$. Lysozyme was also found to correlate with the subscales of PSS, as did SIgA. Except for workload and patient related difficulties, all other PSS subscales were negatively correlated with lysozyme secretion rate. As lysozyme is one of the components contributing to mucosal immunity, the results showed the negative relation between stress and immunity.

As this is a cross sectional study, it is not possible to infer a causal relation between stress and lowered lysozyme levels. However, a possible mechanism that explains the relation is proposed. Psychological stressors have been shown to raise glucocorticoid levels through increased sympathetic activity. An increased level of glucocorticoid is responsible for the observed inhibition of macrophage and monocyte function. ${ }^{34}$ As the macrophage is one of the principal sources of salivary lysozyme, an increase in glucocorticoid level caused by stress can inhibit the production and secretion of lysozyme. ${ }^{19} \mathrm{~A}$ longitudinal study is needed to investigate the relation between lysozyme and stress.

\section{Limitations}

Some limitations of the study should be mentioned. Firstly, the cross sectional research design did not permit us to examine possible sequential relations between self perceived work related stress and the salivary biomarkers. Secondly, although we used a more detailed and specific questionnaire to assess nursing stress, we still could not separate the relative contributions of the individual and the non-working environment when examining the stress response. Longitudinal research that includes not only repeated measures of stress but also related physiological parameters (for example, autonomic nervous system and immunological measures), would provide additional insights into the potential implication of stress induced health impairment.

\section{Conclusions}

ED nurses reported higher levels of stress compared to GW nurses on the PSS. These findings support previous results that ED nursing is more stressful than GW nursing. ED nurses, who perceived a higher level of stress, showed a significantly lower level of salivary IgA and lysozyme than GW nurses. Salivary IgA and lysozyme were inversely correlated with self perceived work related stress. As these salivary biomarkers are reflective of the level of mucosal immunity, results support the inverse relation between work related stress and mucosal immunity found in other studies. 


\section{ACKNOWLEDGEMENTS}

The authors would like to thank Mr BY Low, Ms PG Chan, Mr LS Koh, Ms P Seet, Ms YK Lee, and Mrs A Yew from the respective departments of the hospitals for their support in data collection. We also thank Prof. CN Ong, Mr HY Ong, and all colleagues in the Department of Community, Occupational and Family Medicine in the National University of Singapore for their assistance. Y Yang is supported by a Mobil-NUS postgraduate medical research scholarship of Singapore and a National University of Singapore grant (R-186-000-049-214).

\section{Authors' affiliations}

Y Yang, D Koh, V Ng, G Chan, S E Chia, Dept of Community Occupational, and Family Medicine, Faculty of Medicine, MD3, National University of Singapore, 16 Medical Drive, Singapore 117597 C Y Lee, Emergency Medicine Dept, National University Hospital, 5 Lower Kent Ridge Road, Singapore 119074

F Dong, National University Medical Institute, MD1 1, National University of Singapore, 16 Medical Drive, Singapore 117597 S H Goh, Dept of Accident and Emergency, Changi General Hospital Simei Street 3, Singapore 529889

V Anantharaman, Dept of Emergency Medicine, Singapore General Hospital, Outram Road, Singapore 169608

\section{REFERENCES}

1 Boey KW. Differential relationships of work stress to mental ill health and job dissatisfaction among nursing professionals. Chinese J Psychol $1991 ; 33: 77-86$

2 Wheeler HH. A review of nurse occupational stress research: $1 . \mathrm{Br} J$ Nurs 1997;6:642-5.

3 Walters V, Hains T. Workload and stress in nursing. Can J Nurs Res 1989;21:49-57.

4 Triolo PK. Occupational health hazards of hospital staff nurses. Part I: overview and psychological stressors. AAOHN J 1989;37:232-7.

5 Boey KW, Chan KB, Ko YC, et al. Work stress and psychological well being among the nursing profession in Singapore. Singapore Med J 1997;38:256-60.

6 Dewe PJ. Identifying the cause of nurse's stress: a survey of $\mathrm{New}$ Zealand nurses. Work and Stress 1987;1:15-24.

7 Hipwell AE, Tyler PA. Source of stress and dissatisfaction among nurses in four hospital environments. Br J Med Psychol 1989;62(pt 1):71-9.

8 Keller KL. The management of stress and prevention of burnout in emergency nurses. J Emerg Nurs 1990;16:90-5.

9 Farrington A. Stress and nursing. Br J Nurs 1995;4:574-8.

10 Jemmott JB III, Borysenko JZ, Borysenko $M$, et al. Academic stress, power motivation, and decrease in secretion rate of salivary secretory immunoglobulin Á. Lancet 1983;1:1400-2.

11 Herbert TB, Cohen S. Depression and immunity: a meta-analytic review. Psychol Bull 1993;113:472-86.

12 Evans $\mathbf{P}$, Clow A, Hucklebridge FH. Stress and immune system. Psychologist 1997; 10:303-7.

13 Mestecky J. Saliva as a manifestation of the common mucosal immune system. Ann N Y Acad Sci 1993;694:184-94.
14 Moutsopoulos HM, Karsh J, Wolf RO, et al. Lysozyme determination in parotid saliva from patients with Sjogren's syndrome. Am J Med 1980;69:39-42.

15 Jolles $\mathbf{P}$, Jolles J. What is new in lysozyme research? Mol Cell Biochem 1984;63:165-89.

16 Yeh CK, Dodds MW, Zuo P, et al. A population based study of salivary lysozyme concentrations and candidal counts. Arch Oral Biol 1997:42:25-31.

17 Schenkels LC, Veerman EC, Nieuw Amerongen AV. Biochemical composition of human saliva in relation to other mucosal fluids. Crit Rev Oral Biol Med 1995;6:161-75.

18 Perera S, Uddin, Hayes JA. Salivary lysozyme: a noninvasive marker for the study of the effects of stress on natural immunity. Int J Behav Med 1997; 4:170-8

19 Perera S, Sabin E, Nelson P, et al. Increases in salivary lysozyme and $\lg \mathrm{A}$ concentrations and secretory rates independent of salivary flow rate following viewing of humorous videotape. Int J Behav Med 1998;5:118-28.

$20 \mathrm{Ng} \mathrm{V}$, Koh D, Chan G, et al. Are salivary lgA and lysozyme biomarkers of stress among nurses? J Occup Environ Med 1999:41:920-7.

21 Gasior-Chrzan B, Falk ES. Lysozyme and IgA concentration in serum and saliva from psoriatic patients. Acta Derm Venereol 1992;72:138-40.

22 Cushway D, Tyler PA, Nolan P. Development of a stress scale for mental health professionals. Br J Clin Psychol 1996;35(pt 2):279-95.

23 Guyton AC. Secretory functions of the alimentary tract. In: Textbook of medical physiology, 8th edn. Philadelphia: Saunders, 1991:711.

24 Henningsen GM, Hurrell JJ Jr, Baker F, et al. Measurement of salivary immunoglobulin $A$ as an immunological biomarker of job stress. Scand J Work Environ Health 1992;18(suppl 2): 133-6.

25 Miletic ID, Schiffman SS, Miletic VD, et al. Salivary lgA secretion rate in young and elderly persons. Physiol Behav 1996;60:243-8.

26 SPSS Inc. SPSS for Windows, version 9.0. Chicago: Statistical Products and Service Solutions, 1999

27 Hillhouse JJ, Adler CM. Investigating stress effect patterns in hospital staff nurses: results of a cluster analysis. Soc Sci Med 1997;45:1781-8.

28 Kugler J, Hess M, Hake D. Secretion of salivary immunoglobulin A in relation to age, saliva flow, mood state, secretion of albumin, cortisol, and catecholamines in saliva. J Clin Immunol 1992;12:45-9.

29 Gleeson M, Cripps AW, Clancy RT. Modifiers of the human mucosal immune system. Immunol Cell Biol 1995;73:397-404.

30 Fuhrer R, Stansfeld SA, Chemali J, et al. Gender, social relations and mental health: prospective findings from an occupational cohort (Whitehall II study). Soc Sci Med 1999;48:77-87.

31 Revicki DA, Gershon RM. Work-related stress and psychological distress in emergency medical technicians. J Occup Health Psychol 1996;1:391-6.

32 Hingley P, Cooper CL. Stress and the nurse manager. Chichester: John Wiley \& Sons, 1987.

33 Dunn AJ. Psychoneuroimmunology, stress, and infection. In: Psychoneuroimmunology, stress, and infection. New York: CRC Press, 1996:25-46.

34 Dupont E, Huygen K, Schandene L, et al. Influence of in vivo immunosuppressive drugs on production of lymphokines. Transplantation $1985 ; 39: 143-7$ 